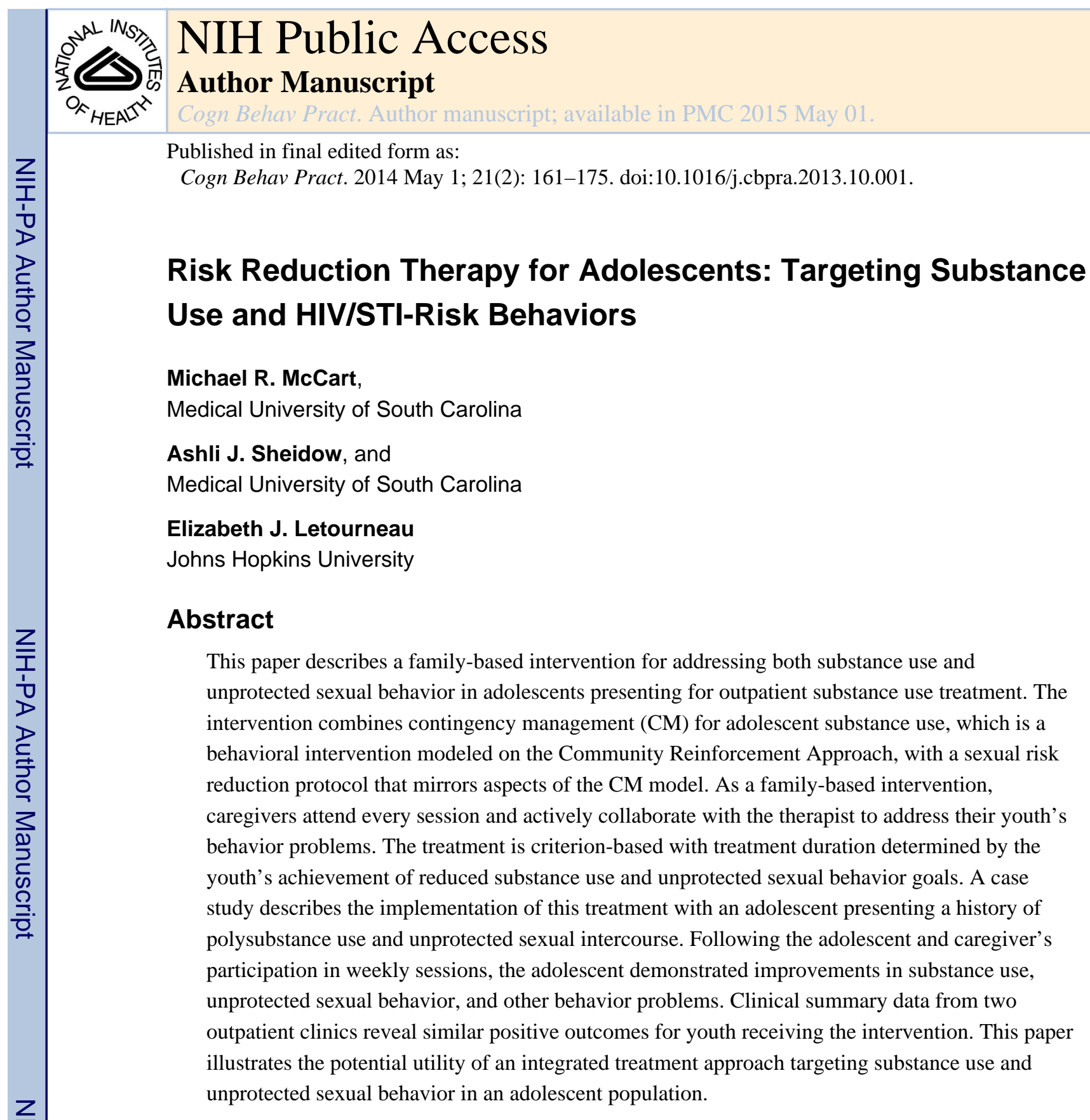

\title{
Keywords
}

adolescents; substance use; HIV/STI risk behavior; HIV/STI prevention

(C) 2013 Association for Behavioral and Cognitive Therapies. Published by Elsevier Ltd.

Address correspondence to: Michael R. McCart, Ph.D., Medical University of South Carolina, 176 Croghan Spur Road, Suite 104, Charleston, South Carolina, 29407; mccartm@musc.edu.

Disclosure Statement

The work reported in this publication was supported by the National Institute on Drug Abuse of the National Institutes of Health under award number R01DA025880. The content is solely the responsibility of the authors and does not necessarily represent the official views of the National Institutes of Health.

Publisher's Disclaimer: This is a PDF file of an unedited manuscript that has been accepted for publication. As a service to our customers we are providing this early version of the manuscript. The manuscript will undergo copyediting, typesetting, and review of the resulting proof before it is published in its final citable form. Please note that during the production process errors may be discovered which could affect the content, and all legal disclaimers that apply to the journal pertain. 
Approximately $40 \%$ of sexually active adolescents in the U.S. report that their last instance of sexual intercourse was unprotected (Centers for Disease Control and Prevention [CDC], 2012a). Unprotected sexual behavior among youth can cause health-related consequences (i.e., HIV/sexually transmitted infections [STIs], unplanned pregnancy), posing tremendous burden for individuals, families, and society. Approximately 2,000 adolescents are diagnosed with HIV each year (CDC, 2010). Epidemiological data also indicate that youth aged 15 to 19 are at disproportionate risk for other STIs. Indeed, individuals in this age cohort represented nearly one third of the 1,412,791 new chlamydia cases and approximately one quarter of the 332,849 new gonorrhea cases documented in 2011 (CDC, 2012b). Adolescents with HIV or other STIs experience elevated rates of physical health, mental health, and social problems (Aral, 2001; Brown et al., 2000; Laufer \& Scott, 2000), and the lifetime medical cost of HIV/STIs among youth in the U.S. exceeds $\$ 6$ billion (Chesson, Blandford, Gift, Tao, \& Irwin, 2004). Regarding pregnancy risk, over 350,000 adolescent girls give birth every year in the U.S. (Martin et al., 2012), and four out of five youth report their pregnancy was unintended (Finer \& Zolna, 2011). Adolescent pregnancy places mothers at risk for preterm labor and places their infants at risk for immediate and long-term health problems (Ventura, Mathews, \& Hamilton, 2001). Further, adolescent childbearing in the U.S. yields costs exceeding \$10 billion each year (Hoffman, 2008).

Adolescents who use or abuse substances are two to eight times more likely to experience an STI or pregnancy relative to their non-substance-using peers (Cavazos-Rehg et al., 2011a; Cook et al., 2006; Staras, Tobler, Maldonado-Molina, \& Cook, 2011; Tapert, Aarons, Sedlar, \& Brown, 2001). This increased STI and pregnancy risk is due, in part, to the elevated rates of unprotected sexual behavior commonly observed among adolescent substance users (Belenko \& Dembo, 2003). For example, compared to non-substance-using adolescents, youth who use drugs or alcohol initiate sexual activity at a younger age and engage in higher rates of unprotected intercourse (Houck et al., 2006; Malow et al., 2006). Among a nationally representative sample of high school seniors, researchers found that the number of self-reported sexual partners steadily increased as substance use intensified from no use to new/experimental use to heavy use (Cavazos-Rehg et al., 2011b). Further, longitudinal research has shown that many substance-using youth continue displaying elevated rates of unprotected sexual behavior into later adolescence and emerging adulthood (Khan, Berger, Wells, \& Cleland, 2012; Strachman, Impett, Henson, \& Pentz, 2009; Wu, Witkiewitz, McMahon, Dodge, \& the Conduct Problems Prevention Research Group, 2010). In this context, leading experts argue that interventions designed to target unprotected sexual behavior among substance-using teens might significantly reduce the burden caused by HIV/STI and pregnancy in this country (Bell et al., 2003; Houck et al., 2006).

Thus, we developed a treatment protocol to simultaneously address substance use and sexual risk behaviors among youth presenting for outpatient substance use treatment. Over the past several years, our group has conducted extensive research on a family-based contingency management $(\mathrm{CM})$ protocol for reducing substance use among adolescents. $\mathrm{CM}$ is a behavioral intervention based on the Community Reinforcement Approach (CRA; Higgins \& Budney, 1993), adapted for use with youth and their families. As with CRA, the CM model utilizes cognitive-behavioral in addition to behavior modification intervention strategies. Decades of research support the effectiveness of these strategies in addressing a 
range of adolescent behavioral problems, including drug and alcohol use (Wolfe \& Mash, 2006). An early version of the CM protocol, developed by Azrin and colleagues, produced promising results in several studies (Azrin et al., 1996; Azrin, Donohue, Besalel, Kogan, \& Acierno, 1994; Donohue \& Azrin, 2001). Subsequent trials conducted by our research team indicated CM improved substance use outcomes among juvenile offenders (Henggeler et al., 2006; Henggeler, McCart, Cunningham, \& Chapman, 2012), and the model is highly amenable to adoption by public sector practitioners (Henggeler, Sheidow, Cunningham, Donohue, \& Ford, 2008; McCart, Henggeler, Chapman, \& Cunningham, 2012).

In addition to reducing substance use, the CM protocol provides a useful framework for targeting the unprotected sexual behavior that places youth at risk for HIV/STI and pregnancy. Thus, we recently developed an intervention integrating evidence-informed sexual risk reduction strategies into the CM platform. The integrated intervention is titled Risk Reduction Therapy for Adolescents (RRTA). Presented here is the first description of RRTA, which we have implemented in outpatient clinic settings for the past 3 years. To illustrate important characteristics of the model, a full case study and summary data on all cases seen in the clinics thus far are presented.

\section{Treatment Approach}

RRTA aims to reduce youth substance use and unprotected sex by targeting two common factors underlying these behaviors: maladaptive parenting and deficiencies in youths' own self-control abilities. Specifically, research indicates that maladaptive parenting (e.g., low supervision, inconsistent discipline, poor communication and affective relations) predicts both substance use (Brown \& Abrantes, 2006) and unprotected sexual behavior (Kotchick, Armistead, \& Forehand, 2006) among youth. Conversely, when caregivers engage in more adaptive parenting strategies, adolescents exhibit reduced substance use (Henggeler et al., 2009; Huey, Henggeler, Brondino, \& Pickrel, 2000) and healthier sexual outcomes, including fewer sexual partners, greater use of contraceptives, and reduced likelihood of STIs (Aspy et al., 2007; Crosby et al., 2006; Perrino, Gonzalez-Soldevilla, Pantin, \& Szapocznik, 2000). A similar pattern of relations exists between adolescent self-control (i.e., the youth's ability to self-monitor behavior, delay gratification, and consider consequences before acting; Wills \& Dishion, 2004) and both substance use and unprotected sex. Indeed, poor self-control is associated with increased risk for substance use (Brody \& $\mathrm{Ge}, 2001$; Wills et al., 2001; Wills, Walker, Mendoza, \& Ainette, 2006) and high-risk sexual behavior (Caspi et al., 1997; Cooper, Wood, Orcutt, \& Albino, 2003; Rosenthal, Muram, Tolley, Peeler, \& Pitts, 1992) among youth, whereas good self-control decreases the likelihood of these outcomes. Thus, to reduce substance use and unprotected sex among adolescents, RRTA uses behavioral and cognitive-behavioral strategies to (a) improve caregivers' parenting skills and (b) build adolescents' self-control abilities. The specific components of RRTA are derived from the aforementioned CM protocol for adolescent substance use, with $\mathrm{CM}$ also providing the platform for strategies that target adolescent sexual risk reduction (SRR; see subsequent description and Figure 1).

RRTA is designed for delivery in an outpatient clinic setting by therapists who hold at least a master's degree in clinical/counseling psychology, social work, or related fields. Sessions 
are held once weekly for 60 to 90 minutes. The treatment is family-based and as such, caregivers are required to attend every session and actively collaborate with therapists in addressing their youth's behavior problems. RRTA also is criterion-based, with treatment duration determined by the youth's achievement of reduced substance use and unprotected sexual behavior goals. In our experience, however, the program is typically completed in 20 to 24 weeks. As noted previously and as described below, RRTA consists of both CM and SRR protocols. The subsequent case study illustrates the protocol components.

\section{Contingency Management (CM)}

The CM portion of RRTA is based on our work implementing this intervention with substance-abusing juvenile offenders (Henggeler et al., 2006; Henggeler, McCart et al., 2012) and training community-based practitioners in CM (Henggeler et al., 2008; McCart et al., 2011). As specified in a published treatment manual (Henggeler, Cunningham, et al., 2012), CM consists of the following core components.

Clinical Assessment-A semistructured clinical interview determines if the youth needs treatment for substance use. Specifically, the therapist gathers information on frequency, quantity, and administration route of the youth's substance use and determines if the use is causing functional impairment. Based on this assessment, the therapist establishes the youth's location on a 4-point continuum that includes (1) experimental use: infrequent use only in the context of recreational activities; (2) early abuse: greater frequency of use with emerging evidence of negative consequences; (3) abuse: frequent use with negative consequences in one or more domains; and (4) dependence: frequent use in spite of multiple negative consequences and considerable activity devoted to seeking and using drugs. Treatment is always recommended for youth at the early abuse, abuse, and dependence points on the continuum. However, for experimental users, additional factors are considered to assess treatment need, including (1) drug of choice: some drugs such as heroin are sufficiently dangerous to merit intervention even if negative consequences have not emerged; (2) youth age: elementary-age youth experimenting with drugs might pose greater risk than teenagers with similar experimental use; (3) acute ingestion: at any age, ingesting large quantities of a drug in a short period is sufficiently risky to merit intervention; and (4) drug use settings: substance use in particularly risky settings (e.g., while driving) might justify intervention. Ultimately, if the therapist observes a link between substance use and negative life outcomes, or potential risk for such outcomes, CM is introduced to the youth and caregiver and the therapist attempts to engage them in treatment.

Antecedent-Behavior-Consequence Assessments-The therapist conducts antecedent-behavior-consequence (ABC) assessments of the youth's substance use in collaboration with the youth and caregiver. In completing the $\mathrm{ABC}$ assessments (similar to behavioral functional analyses), youth are asked to identify environmental, cognitive, and affective triggers (i.e., antecedents) for recent episodes of substance use and to reflect on both the positive and negative consequences of that use.

Self-Management Planning-Triggers identified in the ABC assessments are targeted via self-management plans. Several steps are involved in the development of these plans. 
The process begins by having the youth and caregiver brainstorm a range of potential strategies for dealing with the youth's substance use triggers. Strategies commonly fall into three basic categories, including (a) avoidance of triggers (e.g., taking a different route home from school to avoid a place where drug use commonly occurs, avoiding certain peers), (b) rearranging the environment (e.g., getting rid of items associated with drug use such as pipes and rolling papers, heightened parental monitoring of key times/places of risk), and (c) developing effective drug-refusal skills for unavoidable triggers such as peer pressure. Once a list is generated, the therapist helps the youth and caregiver weigh the potential costs, benefits, and difficulty level of each strategy so they can choose the best plan. Chosen plans are practiced in session via structured role-plays and once the youth becomes proficient with a plan, the youth is assigned homework to try it in real-life situations. Self-management plans are often revisited and refined in session after opportunities for real-life testing.

Drug Testing and Contingency Contracting-Concurrent with the development of self-management plans, the therapist teaches the caregiver how to conduct random urine drug screens (UDS) and breath scans in the home. In addition, screens are conducted at every scheduled treatment session. Finally, the therapist and caregiver arrange for periodic random clinic visits to conduct a screen. Occasionally, urine samples are sent to a lab to verify the results or to determine drug levels. Primarily, though, the instant screens and breath scans are relied upon for monitoring drug use. The therapist, youth, and caregiver create a contingency contract that specifies consequences based on the results of screens.

The contingency contract follows a well-specified protocol (Henggeler, Cunningham, et al., 2012). First, the therapist, youth, and caregiver generate a menu of rewards that can effectively compete with the youth's substance use. The therapist ensures the menu includes a balance between natural incentives the caregiver can provide (e.g., access to cell phone, video games; later curfew; a friend spending the night) and items that can be purchased with gift cards (i.e., therapists have access to $\$ 100$ per adolescent to purchase gift cards from local stores and restaurants). To assist the therapist and caregiver in identifying rewards with the best odds of competing with substance use, rewards are rank ordered by the youth based on preference. From this reward menu, the youth and caregiver choose the youth's "most valued privilege" (or MVP), which is almost always a natural incentive (e.g., access to cell phone, receiving rides from parent, later curfew). Remaining menu items are assigned point values by the caregiver and therapist, usually with each point equivalent to approximately one dollar in value.

Once the menu is finalized, a point-and-level system is implemented and the youth receives a starting balance of 50 points. The youth earns or loses access to the MVP depending on results of weekly drug screens. During the first month (first level of the contingency contract), youth keep their points if they have negative drug screens, but lose 12 points for each week they test positive. Regardless of screen results, youth cannot redeem points in the initial 4-week period. From the fifth week on (second level of the contract), however, negative drug screens result in youth being able to earn additional points and also to use their points to "purchase" items on the reward menu. To provide greater incentive (i.e., escalating reinforcer), the number of points youth can earn each week starts at 12 and increases to 24 after 8 consecutive weeks of negative drug screens (third level of the 
contract). When making a purchase, youth may use as many points as they like from their available balance. Gift cards and other rewards listed on a youth's menu are provided as immediately as possible, consistent with behavioral principles. If a youth has a positive drug screen from the fifth week on, the youth does not earn points and cannot make a purchase with points until the next negative screen. As treatment progresses, emphasis shifts to using natural incentives provided by caregivers to sustain abstinence from drugs and also, if desired by the caregiver, expansion of the behavior plan to include unprotected sexual behaviors and other behaviors the caregiver wishes to target.

\section{Sexual Risk Reduction (SRR)}

The SRR portion of RRTA is also specified in a treatment manual (McCart, Sheidow, \& Letourneau, 2010). This intervention is implemented after CM for two reasons. First, a few key SRR techniques leverage skills and strategies taught to families previously in CM. Second, our experience indicates that therapists, adolescents, and caregivers more comfortably address the youth's sexual risk behaviors after establishing strong engagement during the preceding 3 to 4 months of CM treatment.

The ultimate aim of SRR is to increase safer sexual behaviors, including increased periods of abstinence, decreased number of sexual partners, increased use of protection, and increased HIV/STI testing. Specific SRR techniques were drawn from several primary sources, including a home-based family systems SRR intervention (Letourneau et al., 2013) and federally funded HIV intervention and prevention protocols for Project STYLE (Hadley et al., 2009), Project LIGHT (NIMH Multisite HIV Prevention Trial, 1997), and Project CLEAR (Lightfoot, Rotheram-Borus, \& Tevendale, 2007). The SRR components are described next; the first four components (i.e., clinical assessment, $\mathrm{ABC}$ assessments, behavior planning, and self-management planning) are consistent with strategies from CM (see Figure 1).

Clinical Assessment-The therapist conducts a clinical interview with the youth alone to obtain information on recent (past 3 months) and lifetime history of consensual sexual activity. Questions inquire specifically about the youth's experiences with genital touching (e.g., mutual masturbation) and with oral, anal, and vaginal intercourse. For youth reporting any type of sexual activity, follow-up questions assess how often a barrier form of protection (e.g., condom, dental dam) has been used.

ABC Assessments-If the youth has any history of sexual activity, the therapist and youth meet individually to complete $\mathrm{ABC}$ assessments for recent sexual experiences. As with CM, the goal is to have the youth identify triggers for the problem behavior (i.e., unprotected sex) and abstinence from the problem behavior (i.e., protected sex or declining unprotected sex). Examples of common triggers for unprotected sex include situational contexts (e.g., not having protection available, having a partner who dislikes condoms), cognitions (e.g., thinking sex will feel better without a condom), and affective states (e.g., embarrassment or anxiety). The therapist also has the youth reflect on the positive and negative consequences of past sexual behavior. If the youth has no history of sexual activity, $\mathrm{ABC}$ assessments are not conducted, and the therapist moves straight to the next core 
component. These $\mathrm{ABC}$ assessments are conducted initially with the youth alone to build engagement and trust with the youth. The therapist then prepares the youth to disclose information regarding past sexual behavior to the parent.

Behavior Planning-The therapist collaborates with the youth and caregiver to develop specific rules and consequences for the youth's future sexual behavior. First, the therapist meets individually with the youth and individually with the caregiver to determine any current rules or perceived expectations in the home regarding adolescent dating and sexual behavior. Next, the therapist and caregiver collaborate to specify rules in a written behavior plan. The therapist ensures the caregiver can feasibly measure the adolescent's adherence to all rules. Examples of rules commonly generated by families include requiring that the adolescent introduce a new partner to the caregiver before going on dates, requiring that a responsible adult always be present when the adolescent and partner visit each other's home, and specifying that the adolescent must always have an unexpired condom on hand when going out for the evening. Any failure to adhere to the rules results in a loss of access to the partner for a set period. Once the behavior plan is finalized, the caregiver presents it to the youth in a session. Another goal of this session is for the youth to inform the caregiver about prior sexual experiences. Importantly, the therapist coaches the youth on how to make this disclosure ahead of time.

Self-Management Planning-Next, the therapist, youth, and caregiver develop selfmanagement plans to reduce the youth's sexual risk. For youth with a history of sexual activity, self-management plans target previously identified triggers from the $\mathrm{ABC}$ assessments. For youth without any history of sex, self-management plans are generated for "hypothetical" situations the youth might encounter in the future. Examples of plans commonly generated by families are similar to those generated to address substance use, including (a) avoiding high-risk situations (e.g., being alone at a party with unknown peers), (b) rearranging the environment (e.g., always having condoms available, increased parental monitoring), and (c) use of effective refusal skills (e.g., refusing to engage in consensual sexual behavior unless protection is used). As with CM for substance use, the youth practices with the therapist and caregiver in session prior to trying the plans out in real-life situations.

Education About Sexual Risks and Safety-For this component, the therapist uses structured handouts from the CDC and SAMHSA to educate the youth and caregiver about sexual risks and safety (e.g., http://www.cdc.gov/std/healthcomm/fact_sheets.htm; http:// store.samhsa.gov/product/Tips-for-Teens-The-Truth-About-HIV-AIDS/PHD725).

Regarding sexual risks, the therapist discusses the probability of pregnancy when no protection is used and reviews the signs, symptoms, route of transmission, and treatment method for various STIs (e.g., chlamydia, gonorrhea, bacterial vaginosis, and HIV). The youth and caregiver also receive information on methods for protecting against risk, including abstinence and use of different forms of birth control (e.g., barrier, hormonal, and implantable devices). When reviewing this information, the therapist stresses that while hormonal and implantable birth control methods are highly effective at preventing pregnancy, they do not protect against STIs. Thus, it is emphasized that youth always use 
both hormonal/implantable methods and barrier methods for maximum protection. Information about all forms of birth control is provided, regardless of the youth's gender, and caregivers are guided to take an active role in the discussion.

Referral for HIV/STI Testing-The therapist meets with the youth and caregiver to discuss the importance of HIV/STI testing. Specifically, the therapist explains that youth are at risk for contracting HIV and other STIs whenever they engage in sexual behavior and, because symptoms often do not present for months or years, testing is required to assess for potential infection. Next, the therapist provides information on local HIV/STI testing agencies, including location, hours of operation, and testing fees/procedures. For youth with a history of sex (regardless of whether protection was used), the therapist encourages the youth and caregiver to make a testing appointment for the youth. If they are amenable, the therapist has them schedule an appointment with an agency during the session. The therapist follows up with the youth and caregiver in a later session to ensure they attended the appointment and to problem-solve any barriers to attendance. For youth who test positive for HIV or another STI, the therapist ensures that the youth and caregiver understand and are adhering to treatment.

Condom Use Skill Training-The therapist teaches the youth and caregiver about correct condom usage (i.e., 15 steps) using a model penis. Of note, if an opposite-sex caregiver has been the primary person involved in the sessions, the therapist will first assess whether the youth would feel more comfortable having a same-sex caregiver (or another trusted adult) participate in the training. The therapist meets individually with the caregiver to conduct the condom skill demonstration. Next, the caregiver demonstrates correct condom use to the youth, with assistance from the therapist. Finally, the youth practices the demonstration in session until all of the steps are mastered.

Obtaining Birth Control-Finally, the therapist ensures that the youth and caregiver are knowledgeable regarding local resources to obtain birth control. For both male and female clients, the therapist provides a handout on locations where condoms can be purchased or obtained for free (e.g., state health department clinics, HIV testing centers, Planned Parenthood, stores, and pharmacies). The therapist, youth, and caregiver develop a plan for obtaining condoms, and the youth is instructed to implement the plan as homework. Additional work is conducted with female clients. If the female youth is already using a hormonal or implantable form of birth control, the therapist ensures the youth has regularly scheduled provider visits for medical checkups and prescription refills. If the youth is not using one of these methods, the therapist assists the youth and caregiver in scheduling an appointment with their primary care doctor (or another provider) to learn more about birth control options. At subsequent sessions, the therapist ensures they attended the appointment and problem-solves any barriers.

\section{Case Study of RRTA}

\section{Client Description}

At intake, Zoey (all names changed for confidentiality) was a 16-year-old tenth grader living with her mother, Ms. Rogers, who worked in advertising. Also in the home were Zoey's 
twin brother and 7-year-old sister. The children's father lived out of state and was no longer in touch with the family. Ms. Rogers reported a significant history of alcohol abuse in her extended family, but paternal family history was unknown. Ms. Rogers' primary concerns at intake were Zoey's poor attitude, substance use, and school performance. Zoey was a sophomore in high school. She had Bs in a few classes, but was failing several others. Her goals were to graduate, attend college, and work in web design. She had a history of leaving school to use drugs with friends. Zoey's only extracurricular activity was an art club, which included many of the friends with whom she used drugs. In her free time, Zoey played pool and hung out with friends. She reported all her close friends, including a boyfriend, used drugs. Zoey first used drugs at age 11, but significant use began at age 14. When Zoey was 15 , she ran away from home, lived with some older peers for 2 weeks, and drank daily. She reported periods of daily marijuana use and limited experimentation with cocaine, acid, mushrooms, ecstasy, Xanax, and Ambien. At intake, Zoey reported occasional binge drinking on weekends and smoking marijuana weekly. Her most recent reported use was the week prior to her first RRTA session, and the UDS collected at the intake was positive for marijuana.

Zoey began engaging in sexual intercourse at age 13. She reported vaginal sex with five male partners, including her current boyfriend. She reported having vaginal sex 75 times total, 70 with condoms. She reported giving oral sex to two partners seven times, four of which were protected with condoms. She reported receiving oral sex from her current partner five times, and none of these were protected with a dental dam. She denied anal intercourse. Zoey was arrested with peers for shoplifting and possession of marijuana and alcohol 3 months prior to intake. At the time of arrest, Zoey screened positive for marijuana and alcohol. At intake, she was on probation, with one violation for marijuana use. During treatment, Zoey's twin brother also was arrested for possessing marijuana.

\section{Course of Treatment}

Treatment Introduction and CM Delivery (Sessions 1-8)-Zoey and her mother completed an intake with the RRTA therapist. This assessment identified the need for treatment of both problematic substance use and unprotected sexual behavior. The therapist introduced RRTA, including a brief description of the CM and SRR protocols. Zoey appeared invested in treatment, eager to earn items and trust from her mother and to end probation.

Zoey attended the second session, but her mother did not. The focus of this session was to begin the $\mathrm{ABC}$ assessment component of CM and identify Zoey's triggers for substance use. Zoey's triggers included time with negative peers, access to money, feeling down or bad about herself, having a "hard" day, anger, and boredom. An ABC was completed for alcohol use a few days prior to her session (see Figure 2). Assigned as homework was an ABC assessment for times when Zoey was tempted but did not use substances. No screen was collected since Ms. Rogers was not present, but Zoey self-reported no use. Following the session, the therapist contacted Zoey's mother and discovered Ms. Rogers had misunderstood that she would be needed for each session. Following education on the 
benefits of parental involvement in treatment, Ms. Rogers committed to attending all future sessions.

Zoey and her mother attended the third session together. Zoey's UDS taken in session was negative, and Ms. Rogers reported no suspicions of recent substance use by her daughter. Next, the therapist reviewed Zoey's homework (an ABC for non-use; see Figure 3) and taught Ms. Rogers the elements of the ABC assessment approach. The therapist also taught Ms. Rogers how to conduct home-based instant UDS and breath scans with her daughter, which as noted previously is a standard part of the RRTA intervention. Ms. Rogers requested extra tests so she also could assess Zoey's twin brother, with whom Zoey sometimes used marijuana and alcohol. Ms. Rogers was provided instant breath scans and UDS: mostly 3-panel tests, but also some 6- and 10-panel tests to assess for a broad range of substances. Ms. Rogers was instructed to complete UDS with Zoey and her brother randomly at least every 4 days, breath scans at random but especially on weekends, and random room sweeps to search for drug use paraphernalia. Ms. Rogers also was asked to report the results of Zoey's home-based screens to the therapist via phone.

Access to money was identified as a primary trigger for Zoey's marijuana use. Thus, the fourth session focused on developing an initial self-management plan that resulted in Ms. Rogers limiting Zoey's access to money. The therapist also reviewed results from the home and session screens (all were negative) and completed new ABC assessments for Zoey's non-use of substances.

The contingency contract was presented at the fifth session, and Zoey identified potential rewards (a pet ferret, new clothes and jewelry, access to the family car, driving classes, a favorite meal, having a friend spend the night, a family day trip to the beach, regained access to her iPod Touch, and earning her driver's license). The therapist reviewed Zoey's potential rewards menu separately with Ms. Rogers. Ms. Rogers approved all items, and she agreed to one of Zoey's choices for MVP, which was access to her iPod Touch. Zoey was also excited about earning a ferret and new clothes, so the greatest point values were placed on these items. Regarding access to the family car, it was decided that Zoey could earn this reward only after 8 consecutive weeks of negative drug screens (i.e., the third level of the contingency contract) since Zoey's probation officer requested this reward not be given until Zoey showed evidence of sustained abstinence. Ms. Rogers presented the final reward menu to Zoey. Because Zoey's session and home screens were negative for a fifth straight week, Zoey was permitted to make a purchase with her points, but she opted to continue accruing them for a larger reward. All of the screens conducted with Zoey for the remainder of treatment were negative.

At Sessions 6 through 8, additional self-management plans were developed to address Zoey's triggers for substance use (e.g., boredom, running into a friend with whom she used to smoke marijuana). Zoey and Ms. Rogers identified and evaluated the benefits and costs for several plans to address these triggers, choosing the best (e.g., going to an approved friend's house, working, exercising with Ms. Rogers, going on a bike ride, or drawing when bored). They were not willing to pursue other prosocial activities to reduce boredom since Zoey had recently started a part-time job at a local restaurant. Zoey and Ms. Rogers role-

Cogn Behav Pract. Author manuscript; available in PMC 2015 May 01. 
played the chosen self-management plans. The therapist also assisted Zoey in selecting effective drug-refusal skills as part of self-management planning. As a short-term strategy, Zoey decided she would tell her friends that she could not use substances because she was on probation. Her long-term plan included telling peers that she "got off probation and does not want to go back." Zoey felt $100 \%$ confident these excuses would work with her peers and role-played them with Ms. Rogers. Homework was assigned to practice these selfmanagement plans in real-life settings.

Delivery of SRR Components (Sessions 9-13)-The therapist introduced SRR to Zoey and Ms. Rogers in Session 9. Following the introduction, the therapist met individually with Zoey to review her past sexual experiences and triggers for unprotected sex practices. Zoey had reported using condoms for most instances of vaginal sex and in some instances of giving but not receiving oral sex. Further, she did not use birth control other than condoms. Triggers for inconsistent protection identified in $\mathrm{ABC}$ assessments included not having condoms, not thinking to use one, and not being aware of how to use a dental dam during receptive oral sex. Barriers to oral contraceptives included Zoey's attribution that "the pill" caused her to gain weight in the past. Zoey stated she had previously informed her mother about her experiences with vaginal sex. However, Zoey reportedly told Ms. Rogers that she had always used a condom (note, the therapist helps Zoey correct this misinformation in a later session with Ms. Rogers). Next, the therapist met with Zoey and Ms. Rogers together to describe the remaining aspects of the SRR intervention. Toward the end of the session, Zoey asked if she could take a few condoms from a jar on the therapist's desk. Ms. Rogers approved this request, but asked that the therapist also review additional methods of contraception. Therefore, the component on obtaining birth control was briefly introduced. Ms. Rogers and Zoey ultimately decided they would schedule an appointment with their family gynecologist so Zoey could learn more about hormonal and implantable forms of contraception. Of note, Ms. Rogers stated she was unable to complete a home-based UDS over the week because Zoey's brother took all of the screens to test himself prior to a court appearance. Thus, it was suggested that Ms. Rogers store the UDS in a lockbox, which was provided by the therapist.

Birth control options were reviewed more thoroughly in Session 10, and the therapist asked whether Zoey and Ms. Rogers had met with their gynecologist. Ms. Rogers reported she had not yet scheduled the appointment, but committed to calling later in the day and put a reminder in her phone. An assessment of family conversations and expectations about dating and sex was completed individually with Zoey and Ms. Rogers. Zoey described a number of her mother's expectations (see Table 1). Ms. Rogers, on the other hand, reported only a few expectations to the therapist (see Table 1). As none of the expectations listed by Ms. Rogers specifically addressed sexual behavior, the therapist asked Ms. Rogers to clarify these expectations. According to Ms. Rogers, she expected Zoey to be selective about her sexual partners (e.g., only have sex with someone who is well-known and trusted by both Zoey and Ms. Rogers) and she did not want Zoey having sex in her home. Ms. Rogers also stated that she had told Zoey to always use condoms, and she was recently surprised to learn her daughter had stopped taking "the pill" due to concerns about weight gain. As homework, Ms. Rogers was asked to decide on a final set of rules to include in a behavior plan contract. 
In anticipation for the end of school, time was spent in Session 11 discussing a summer monitoring and supervision plan. In addition, the therapist discussed strategies Zoey could use to prevent boredom (a previously identified substance use trigger). Ms. Rogers decided to conduct more frequent home-based drug screens and agreed to drop by the house randomly during the work day to check on her daughter. Zoey planned to increase her work schedule to 5 days per week, and the family identified a photography class for Zoey at a local technical college. Next, the therapist individually prepared Zoey for a conversation with her mother about past sexual experiences and the establishment of rules on sex and dating behavior. The therapist also met individually with Ms. Rogers to finalize rules for the revised behavior plan, which would be presented to Zoey at the next session. The rules specified by Ms. Rogers are listed in Table 1. An ABC assessment for unprotected sex was assigned as homework, and Ms. Rogers confirmed that a gynecological appointment had been scheduled for her daughter.

During Session 12, the therapist facilitated a conversation between Zoey and Ms. Rogers that involved Zoey's disclosure of past unprotected sex and Ms. Roger's presentation of the revised behavior plan contact. In addition, Zoey's unprotected sex ABC assessment was reviewed. Zoey's unprotected sex "triggers" included not having a condom on hand, not developing a plan for negotiating condom use, and believing that her partner was "safe" (i.e., free of STIs) because he was a "trusted" friend. The family was instructed to start using the revised behavior plan, which now included earning/removing privileges based on dating and sexual behaviors. Of note, by this point in treatment, Zoey had accrued several points based on negative drug screens, and she decided to "purchase" her driver's license from the reward menu.

Shortly following Session 12, Ms. Rogers called to report that the gynecological visit had occurred, but Zoey did not get a prescription for contraception because she reported feeling "uncomfortable" with the gynecologist. A few days later, Ms. Rogers called the therapist again to report that an STI test conducted by the gynecologist had just come back positive for chlamydia. Ms. Rogers stated Zoey was "mortified" by the results, and Zoey wanted to avoid seeing this particular gynecologist again. The therapist agreed to hold an emergency session with Zoey and Ms. Rogers the next day.

At Session 13, the therapist met individually with Zoey to discuss the chlamydia test result. Zoey reported that she contracted chlamydia from her boyfriend after having unprotected vaginal sex. She stated that tests for HIV and other STIs were negative. According to Zoey, she engaged in unprotected sex because her boyfriend stated he had recently been tested for STIs and was "clean." Zoey told the therapist that she decided to "dump" her boyfriend because he had lied. The therapist and Zoey completed an ABC assessment on unprotected sex and developed a self-management plan based on identified triggers. Ms. Rogers then joined the session, and the RRTA education about sexual risk component was provided. Zoey reported she had decided to return to the same gynecologist, had scheduled a follow-up appointment, was taking medication for chlamydia, and was practicing abstinence until she tested negative for the STI. Thus, although outside of the typical RRTA protocol sequence, the component for HIV/STI testing was achieved at this point. 
Management of Behavioral Slips (Sessions 14-17)—During Session 14, Zoey's behavior at home was discussed as it presented a barrier to her receiving rewards from her reward menu. Ms. Rogers reported that although Zoey continued to accrue points for negative screens, she was reluctant to allow use of these points because Zoey had started "talking back" to her mother on a regular basis. The therapist met individually with Ms. Rogers to determine if the contract should be revised to incorporate this new problem behavior. One presented option was to combine drug use and "talking back" into a single category whereby abstinence from both behaviors was required to receive access to rewards. Ms. Rogers ultimately decided against combining these behaviors, and instead chose to provide Zoey with bonus points each day that she did not "talk back." After presenting this change to Zoey, the therapist conducted condom use skill training, as described previously in the Treatment Approach section.

The next scheduled session was missed because Zoey ran away from home. After 3 days away from home and school, Zoey returned home and attended her 15th appointment. According to Zoey, she was failing several classes and decided to run away and hang out with friends instead of facing the problem. All results from UDS and breath scans were negative, despite Zoey's decision to run away. Zoey's probation officer initially suggested detention as a consequence, but the therapist and Ms. Rogers requested permission to try a home-based plan first. The officer relented but ordered weekly random UDS to be collected by probation. The therapist, Ms. Rogers, and Zoey developed plans to ensure Zoey attended school and performed better in her classes. Plans also were developed to improve Ms. Rogers' ability to monitor Zoey's attendance and school performance. Ms. Rogers decided to establish daily email correspondence with Zoey's teachers to track these behaviors. An $\mathrm{ABC}$ assessment on abstaining from drugs while away from home was assigned as homework.

At Session 16, Ms. Rogers expressed concern that the new behavior plan was not effective because Zoey was not attending all of her classes and had not completed her missed assignments. The therapist met individually with Ms. Rogers and a decision was made to tie Zoey's school behaviors to a distinct, meaningful MVP. Specifically, while Zoey would continue to receive her MVP for drug abstinence, she could also earn a separate MVP for attendance at all classes and completion of all assignments in a given week. Ms. Rogers presented this change to Zoey in session, and time was also spent reviewing Zoey's homework assignment from the previous week. Finally, the obtaining condoms component from the SRR protocol was completed, and homework was assigned for Zoey to obtain condoms at a location near her home.

Based on Ms. Rogers' request, Session 17 focused on amending the reward menu to include more meaningful incentives. Zoey was able to add driving (with Ms. Rogers in the car) to her menu, and she could purchase a gift card for clothes with her points. She also added driving alone to the menu, with a requirement of four consecutive weeks of school attendance. Ms. Rogers reported the need to reschedule the follow-up chlamydia test. Selfmanagement plans were developed and reviewed for having sex with a trusted partner (see Figure 4) and for not having condoms. 
Completion and Discharge (Sessions 18-20)-At Session 18, Zoey and Ms. Rogers stated that the gynecological visit had been rescheduled for the following week, and they added reminders in their mobile phones. They reported $100 \%$ school attendance and said Zoey had turned in all missed assignments and was completing make-up work to improve her grades. Based on her drug abstinence and improved school attendance/performance, Zoey was moved to the final phase of probation, which required only monthly meetings with her probation officer. Remaining birth control options were reviewed in session, and it was decided Zoey would consult with her gynecologist on a potential method to try. Zoey also earned her Drug Screen MVP and her School Performance/Attendance MVP.

At Session 19, Zoey reported she had attended her gynecological appointment and tested negative for chlamydia. She also obtained a prescription for "the patch" and a schedule for starting this birth control method. Zoey reported continuing to abstain from sex to avoid pregnancy and STIs. The therapist, Ms. Rogers, and Zoey discussed strategies for effectively refusing unprotected sex. Options ranged from always having a condom (Zoey rated this easiest) to telling the potential partner sex could occur next time they saw one another if he had condoms (rated second easiest) to using an excuse such as menstruation or pretending her mother called demanding she come home immediately (rated third easiest). Zoey and Ms. Rogers role-played each of these strategies in session. Options discarded as too difficult were just saying no and giving the rationale that she could get STIs or get pregnant.

The final session focused on praising Zoey for having negative UDS and breath scans for the past 6 months and actively participating in the risk reduction program. The therapist presented two small rewards and a certificate of achievement to Zoey. Long-term plans were discussed with Ms. Rogers to continue the behavior plan, including ongoing drug testing after discharge from treatment. At the close of treatment, Zoey was rated as not having any clinically significant symptoms (see below description).

\section{Clinical Data Summary}

RRTA has been conducted in two outpatient clinics, with extensive training and fidelity monitoring by the developers. Therapists have rated engagement and outcomes for the 50 families who have received it thus far. After discharge (either treatment completion or dropout), therapists complete ratings of client and family engagement on a 4-point scale (high, medium, low, none) and of 13 youth symptoms using an adapted version of the Target Symptom Rating (Barber, Neese, Coyne, Fultz, \& Fonagy, 2002). Symptoms are rated with 5-point scales that vary by item. For substance use, rating descriptions included: (a) no substance use; (b) mild substance use, e.g., infrequent use with no adverse consequences or craving; (c) moderate substance use, e.g., occasional use at parties or in other social situations, intending to continue at this level; (d) serious substance use, e.g., frequent use with one or more adverse effects; (e) severe substance use, e.g., frequent use with multiple adverse effects. Sexual risk behaviors were defined as: (a) no high-risk sexual behaviors/ abstinent; (b) mild sexual risk behaviors, e.g., infrequent sex and always uses protection, no casual sex or one-night-stands; (c) moderately risky behaviors, e.g., unprotected sex on occasion, sex with just one or two partners but usually uses protection, no casual sex or onenight-stands; (d) serious risky behaviors, e.g., frequent and often unprotected sex, multiple 
partners, some casual sex or one-night-stands; (e) severe risky behaviors, e.g., prostitution, high-risk partners, no use of protection, frequent casual sex or one-night-stands. Other symptoms and problem areas rated were depression, anxiety, psychoticism, family conflict, peer relationship problems, school difficulties, acting-out/criminal behaviors, aggression, suicidality, psychosomaticism, and impulsivity, with ratings ranging from $1=$ no symptoms/ concerns to $5=$ severe symptoms/concerns.

Engagement was high or medium for most families, although one-fifth to one-quarter had low engagement (see Table 2). This positive engagement was reflected in high session attendance. In fact, most families missed no more than 2 sessions (median) and the modal number of missed sessions was 1 . The two symptom ratings addressing primary outcomes of RRTA included substance use and sexual risk behaviors. At the end of treatment, the majority of youth were rated as having no substance use (see Table 3), with only three having moderate use (4\%) or serious use (2\%) and none having severe use. With respect to sexual risk behaviors, the majority of youth were rated as having no or mild sexual risk behaviors, with one-fifth having moderate risk, only one having serious risk, and none having severe risk (see Table 3). Further, no youth became pregnant or impregnated partners during treatment. Sexual risk behaviors were unknown for four youth whose treatment ended prior to completion of RRTA. For the 11 remaining symptoms, most cases were rated as having no problems, ranging from $56 \%$ having no family conflict to $100 \%$ having no psychotic symptoms. Few to no cases had serious or severe levels of these symptoms.

\section{Conclusion}

Substance-using adolescents are at disproportionate risk for HIV/STI owing to their elevated involvement in unprotected sexual behavior. Adolescent substance use treatment, therefore, represents an ideal opportunity to reduce HIV/STI risk through interventions promoting safety in adolescents' sexual relationships. RRTA is an example of an integrated protocol designed to address both substance use and unprotected sexual behavior among youth presenting for outpatient substance use treatment. Our experience implementing RRTA over the last few years suggests this treatment can yield positive outcomes with regard to substance use, unprotected sexual behavior, and other clinical problems. It is important to acknowledge, however, that these outcomes were achieved in the context of a rigorous quality assurance protocol. Specifically, all therapists were required to participate in a 5-day RRTA training before implementing the intervention. In addition, the first author (a licensed clinical psychologist) routinely reviewed the therapists' audiotaped RRTA sessions and provided them with weekly individual supervision. Finally, on a bi-annual basis, booster trainings were conducted to promote therapist competence in intervention delivery. Future work would be needed to determine if similarly positive outcomes could be achieved with less intensive training and supervision procedures.

As expected, RRTA therapists occasionally encountered challenges when implementing the intervention with families. A few challenges are described next, along with strategies we have used to address the problems. First, caregivers sometimes questioned the need for an intervention that promotes such intensive parenting, noting adolescents should be old enough to make the "right" decisions. In these situations, therapists explain that while 
adolescents are often adult-like in appearance, aspects of the brain (frontal lobes) governing judgment, insight, and rational decision-making are not fully developed until much later in life. As a result, caregivers play an important role in providing their adolescents with ongoing guidance, monitoring, and support. Further, therapists note that peer influence represents one of the strongest risk factors for adolescent substance use and unprotected sex. Adolescents are more likely to associate with negative peers when there is a lack of close caregiver supervision. Conversely, research shows that when caregivers monitor adolescents and set clear rules about behavior, the likelihood of substance use and unprotected sex decreases. Thus, it is stressed to caregivers that their involvement in RRTA is critical for achieving and maintaining good clinical outcomes.

As a second challenge, therapists sometimes learn the youth's caregiver has his or her own substance use problem. When this occurs, the therapist meets individually with the caregiver to express concern about how this substance use might impact the youth's treatment. RRTA therapists encourage these caregivers to obtain their own substance use treatment, and make referrals as needed. In addition, the therapist tries to anticipate how the caregiver's substance use might be a barrier for the youth. For example, if the scent of marijuana is a trigger for the youth, explicit plans are developed so the caregiver avoids smoking marijuana in the house. If, despite these efforts, the caregiver's substance use continues to interfere with the youth's treatment, the therapist and caregiver determine if there are nonusing adult family members who could attend the sessions and assist in implementing the RRTA interventions. Overall, the goal is to support the caregiver in his or her role as a parent, while at the same ensuring any barriers to the youth's treatment are effectively addressed.

A third challenge involves situations where caregivers demand abstinence with youth who are already sexually active. In our experience, this is a relatively infrequent occurrence. Indeed, although all caregivers want youth to behave safely and responsibly, they rarely expect youth to completely forego sexual activity once initiated. Nevertheless, if a caregiver indicates abstinence is the goal, the therapist helps prepare the caregiver for communicating this goal to the youth in a family session. The caregiver is encouraged to express his or her reasons for wanting the youth to remain abstinent, while also acknowledging that the youth's sexual urges are developmentally normative. In addition, caregivers express the desire for youth to talk with them first before engaging in future sexual activity. The ultimate aim is for caregivers to openly express the abstinence goal while also sending the message that youth can come to the caregiver with questions about safety if and when a decision is made to have sex.

Some limitations of our work should be noted. First, our clinical outcome data were derived solely from self-reports and therapist observations and therefore should be interpreted with caution. Second, while it is expected that youth in our sample would enter treatment with high rates of substance use and possibly sexual risk, the posttreatment data for the other psychosocial variables in Table 2 are less informative without pretreatment values for comparison. Third, the information presented here is based on our clinical experience implementing RRTA, and the treatment needs to be formally evaluated in a randomized efficacy trial. Fortunately, such a trial is currently under way. Importantly, the ongoing study is using a multimethod approach to longitudinally measure youth substance use, sexual risk, 
and other clinical problems (e.g., delinquent offending, mental health symptoms), which will ultimately help address the measurement limitations noted previously. If results from the efficacy trial are favorable, future work will involve evaluating RRTA in a larger effectiveness study. In addition, dismantling research might ultimately be needed to examine the relative effectiveness of the various RRTA intervention components. In sum, our experience implementing RRTA thus far has been promising and, with a focus on both substance use and unprotected sexual behavior outcomes, interventions such as RRTA might represent a powerful tool for improving the long-term health outcomes of at-risk youth.

\section{Acknowledgments}

The authors would like to thank Sarah B. Hales, LMSW, for her contributions to the development of various RRTA implementation and training materials.

\section{References}

Aral SO. Sexually transmitted diseases: Magnitude, determinants and consequences. International Journal of STD and AIDS. 2001; 12:211-215. [PubMed: 11319969]

Aspy C, Vesely SK, Oman RF, Rodine S, Marshall L, McLeroy K. Parental communication and youth sexual behavior. Journal of Adolescence. 2007; 30:449-466. [PubMed: 16750265]

Azrin NH, Acierno R, Kogan E, Donohue B, Besalel V, McMahon PT. Follow-up results of supportive versus behavioral therapy for illicit drug abuse. Behavioral Research and Therapy. 1996; 34:41-46.

Azrin NH, Donohue B, Besalel VA, Kogan ES, Acierno R. Youth drug abuse treatment: A controlled outcome study. Journal of Child and Adolescent Substance Abuse. 1994; 3:1-16.

Barber CC, Neese DT, Coyne L, Fultz J, Fonagy P. The Target Symptom Rating: A brief clinical measure of acute psychiatric symptoms in children and adolescents. Journal of Clinical Child and Adolescent Psychology. 2002; 31:181-192. [PubMed: 12056102]

Belenko S, Dembo R. Treating adolescent substance abuse problems in the juvenile drug court. International Journal of Law and Psychiatry. 2003; 26:87-110. [PubMed: 12554002]

Bell DN, Martinez J, Gotwinick G, Shaw K, Walker LE, Dodds S, Siciliano C. Case finding for HIVpositive youth: A special type of hidden population. Journal of Adolescent Health. 2003; 33:10-22. [PubMed: 12888283]

Brody GH, Ge X. Linking parenting processes and self-regulation to psychological functioning and alcohol use during early adolescence. Journal of Family Psychology. 2001; 15:82-94. [PubMed: 11322087]

Brown, SA.; Abrantes, AM. Substance use disorders. In: Wolfe, DA.; Mash, EJ., editors. Behavioral and emotional disorders in adolescents: Nature, assessment, and treatment. New York, NY: Guilford; 2006. p. 226-256.

Brown LK, Schultz JR, Parsons JT, Butler RB, Forsberg AD, Kocik SM, Aledort L. Sexual behavior change among human immunodeficiency virus-infected adolescents with hemophilia. Pediatrics. 2000; 106:E22. [PubMed: 10920178]

Budney, AJ.; Higgins, ST. A community reinforcement plus vouchers approach: Treating cocaine addiction (NIH Pub. No. 98-4309). Rockville, MD: National Institute on Drug Abuse; 1998.

Caspi A, Begg D, Dickson N, Harrington H, Langley J, Moffitt TE, Silva PA. Personality differences predict health-risk behaviors in young adulthood: Evidence from a longitudinal study. Journal of Personality and Social Psychology. 1997; 73:1052-1063. [PubMed: 9364760]

Cavazos-Rehg PA, Krauss MJ, Spitznagel EL, Schootman M, Cottler LB, Bierut LJ. Substance use and the risk for sexual intercourse with and without a history of teenage pregnancy among adolescent females. Journal of Studies on Alcohol and Drugs. 2011a; 72:194-198. [PubMed: 21388592] 
Cavazos-Rehg PA, Krauss MJ, Spitznagel EL, Schootman M, Cottler LB, Bierut LJ. Number of sexual partners and association with initiation and intensity of substance use. AIDS and Behavior. 2011b; 15:869-874. [PubMed: 20107887]

Centers for Disease Control and Prevention. Diagnoses of HIV infection and AIDS in the United States and Dependent Areas, 2010. HIV Surveillance Report. 2010; 20 Retrieved from http:// www.cdc.gov/hiv/surveillance/resources/reports/2010report.

Centers for Disease Control and Prevention. Youth Risk Behavior Surveillance United States, 2011. Morbidity and Mortality Weekly Report. 2012a; 61 Retrieved from http://www.cdc.gov/ HealthyYouth/yrbs/index.htm.

Centers for Disease Control and Prevention. Sexually Transmitted Disease Surveillance 2011. Atlanta, GA: US Department of Health and Human Services; 2012b. Retrieved from http:// www.cdc.gov/std/stats11/default.htm

Chesson HW, Blandford JM, Gift TL, Tao G, Irwin KL. The estimated direct medical cost of sexually transmitted diseases among American youth, 2000. Perspectives on Sexual and Reproductive Health. 2004; 36:11-19. [PubMed: 14982672]

Cook RL, Comer DM, Wiesenfield HC, Chang CC, Tarter R, Lave JR, Clark DB. Alcohol and drug use and related disorders: An unrecognized health issue among adolescents and young adults attending sexually transmitted disease clinics. Sexually Transmitted Diseases. 2006; 33:565-570. [PubMed: 16572042]

Cooper ML, Wood PK, Orcutt HK, Albino A. Personality and the predisposition to engage in risky or problem behaviors during adolescence. Journal of Personality and Social Psychology. 2003; 84:390-410. [PubMed: 12585812]

Crosby R, Voisin D, Salazar LF, DiClemente RJ, Yarber WL, Caliendo AM. Family influences and biologically confirmed sexually transmitted infections among detained adolescents. American Journal of Orthopsychiatry. 2006; 76:389-394. [PubMed: 16981818]

Donohue, B.; Azrin, NH. Family behavior therapy. In: Wagner, EF.; Waldron, HB., editors. Innovations in adolescent substance abuse interventions. New York, NY: Pergamon Press; 2001. p. 205-227.

Finer LB, Zolna MR. Unintended pregnancy in the United States: Incidence and disparities, 2006. Contraception. 2011; 84:478-485. [PubMed: 22018121]

Hadley W, Brown LK, Lescano CM, Kell H, Spalding K, Diclemente R. Project STYLE Study Team. Parent-adolescent sexual communication: Associations of condom use with condom discussions. AIDS and Behavior. 2009; 13:997-1004. [PubMed: 18841462]

Henggeler, SW.; Cunningham, PB.; Rowland, MD.; Schoenwald, SK.; Swenson, CC.; Sheidow, AJ.; Randall, J. Contingency management for adolescent substance abuse: A practitioner's guide. New York, NY: Guilford Press; 2012.

Henggeler SW, Halliday-Boykins CA, Cunningham PB, Randall J, Shapiro SB, Chapman JE. Juvenile drug court: Enhancing outcomes by integrating evidence-based treatments. Journal of Consulting and Clinical Psychology. 2006; 74:42-54. [PubMed: 16551142]

Henggeler SW, Letourneau EJ, Chapman JE, Borduin CM, Schewe PA, McCart MR. Mediators of change for multisystemic therapy with juvenile sexual offenders. Journal of Consulting and Clinical Psychology. 2009; 77:451. [PubMed: 19485587]

Henggeler SW, McCart MR, Cunningham PB, Chapman JE. Enhancing the effectiveness of juvenile drug courts by integrating evidence-based practices. Journal of Consulting and Clinical Psychology. 2012; 80:264-275. [PubMed: 22309470]

Henggeler SW, Sheidow AJ, Cunningham PB, Donohue BC, Ford JD. Promoting the implementation of an evidence-based intervention for adolescent marijuana abuse in community settings: Testing the use of intensive quality assurance. Journal of Clinical Child and Adolescent Psychology. 2008; 37:682-689. [PubMed: 18645758]

Higgins, ST.; Budney, AJ. Treatment of cocaine dependence through the principles of behavior analysis and behavioral pharmacology. In: Onken, LS.; Blaine, JD.; Boren, JJ., editors. Behavioral treatments for drug abuse and dependence: National Institute on Drug Abuse Research Monograph 137. Rockville, MD: National Institutes of Health; 1993. p. 97-121.NIH Publication No. 93-3684 
Houck CD, Lescano CM, Brown LK, Tolou-Shams M, Thompson J, DiClemente R, Fernandez MI. Project-SHIELD Study Group. Islands of risk: Subgroups of adolescents at risk for HIV. Journal of Pediatric Psychology. 2006; 31:619-629. [PubMed: 16120764]

Hoffman, S. Counting it up: The public costs of teen childbearing. Washington, DC: National Campaign to Prevent Teen and Unplanned Pregnancy; 2008. Retrieved from http:// www.thenationalcampaign.org/costs

Huey SJ Jr, Henggeler SW, Brondino MJ, Pickrel SG. Mechanisms of change in Multisystemic Therapy: Reducing delinquent behavior through therapist adherence, and improved family and peer functioning. Journal of Consulting and Clinical Psychology. 2000; 68:451-467. [PubMed: 10883562]

Khan MR, Berger AT, Wells BE, Cleland CM. Longitudinal associations between adolescent alcohol use and adulthood sexual risk behavior and sexually transmitted infection in the United States: Assessment of differences by race. American Journal of Public Health. 2012; 102:867-876. [PubMed: 22493999]

Kotchick, BA.; Armistead, L.; Forehand, RL. Sexual risk behavior. In: Wolfe, DA.; Mash, EJ., editors. Behavioral and emotional disorders in adolescents: Nature, assessment, and treatment. New York, NY: Guilford; 2006. p. 563-588.

Laufer M, Scott GB. Medical management of HIV disease in children. Pediatric Clinics of North America. 2000; 47:127-153. [PubMed: 10697645]

Letourneau EJ, Ellis DA, Naar-King S, Chapman JE, Cunningham PB, Fowler S. Multisystemic therapy for poorly adherent youth with HIV: Results from a pilot randomized controlled trial. AIDS Care. 2013; 25:507-514. [PubMed: 22909294]

Lightfoot M, Rotheram-Borus MJ, Tevendale H. An HIV-preventive intervention for youth living with HIV. Behavior Modification. 2007; 31:345-63. [PubMed: 17438347]

Malow RM, Déveiux JG, Rosenberg R, Samuels DM, Jean-Gilles MM. Alcohol use severity and HIV sexual risk among juvenile offenders. Substance Use and Misuse. 2006; 41:1769-1788. [PubMed: 17118815]

Martin JA, Hamilton BE, Ventura SJ, Osterman MJK, Wilson EC, Mathews TJ. Births: final data for 2010. National Vital Statistics Report. 2012; 61:1-72.

McCart MR, Henggeler SW, Chapman JE, Cunningham PB. System-level effects of integrating a promising treatment into juvenile drug courts. Journal of Substance Abuse Treatment. 2012; 43:231-243. [PubMed: 22154039]

McCart, MR.; Sheidow, AJ.; Letourneau, EJ. Risk reduction therapy for adolescents. Department of Psychiatry and Behavioral Sciences, Medical University of South Carolina; 2010. Unpublished treatment manual

NIMH Multisite HIV Prevention Trial. AIDS. 1997; 11:S13-S19. [PubMed: 9475707]

Perrino T, Gonzalez-Soldevilla A, Pantin H, Szapocznik J. The role of families in adolescent HIV prevention: A review. Clinical Child and Family Psychology Review. 2000; 3:81-96. [PubMed: 11227063]

Rosenthal T, Muram D, Tolley E, Peeler M, Pitts B. Teenage pregnancy: Predicting the adolescent at risk. Journal of Sex Education and Therapy. 1992; 18:277-285.

Staras SAS, Tobler AL, Maldonado-Molina MM, Cook RL. Riskier sexual partners contribute to the increased rate of sexually transmitted diseases among youth with substance use disorders. Sexually Transmitted Diseases. 2011; 38:413-418. [PubMed: 21139514]

Strachman A, Impett EA, Henson JM, Pentz MA. Early adolescent alcohol use and sexual experience by emerging adulthood: A 10-year longitudinal investigation. Journal of Adolescent Health. 2009; 45:478-482. [PubMed: 19837354]

Tapert SF, Aarons GA, Sedlar GR, Brown SA. Adolescent substance use and sexual risk-taking behavior. Journal of Adolescent Health. 2001; 28:181-189. [PubMed: 11226840]

Ventura SJ, Mathews TJ, Hamilton BE. Birth to teenagers in the United States, 1940-2000. Vital Health Statistics Report. 2001; 49:1-30.

Wills TA, Cleary SD, Filer M, Shinar O, Mariani J, Spera K. Temperament related to early-onset substance use: Test of a developmental model. Prevention Science. 2001; 2:145-163. [PubMed: $11678291]$ 
Wills TA, Dishion TJ. Temperament and adolescent substance use: A transactional analysis of emerging self-control. Journal of Clinical Child and Adolescent Psychology. 2004; 33:69-81. [PubMed: 15028542]

Wills TA, Walker C, Mendoza D, Ainette MG. Behavioral and emotional self-control: Relations to substance use in samples of middle and high school students. Psychology of Addictive Behaviors. 2006; 20:265-278. [PubMed: 16938064]

Wolfe, DA.; Mash, EJ. Behavioral and emotional disorders in adolescents: Nature, assessment, and treatment. New York, NY: Guilford; 2006.

Wu J, Witkiewitz K, McMahon RJ, Dodge KA. the Conduct Problems Prevention Research Group. A parallel process growth mixture model of conduct problems and substance use with risky sexual behavior. Drug and Alcohol Dependence. 2010; 111:207-214. [PubMed: 20558013] 


\section{Highlights}

- CBT protocol for substance use and unprotected sexual behavior among adolescents presenting for substance use treatment.

- A case study is used to illustrate implementation of the treatment protocol with an adolescent client.

- Our experience implementing the treatment protocol suggests that it can yield positive outcomes among youth.

- Efforts are under way to evaluate the protocol in a randomized trial. 


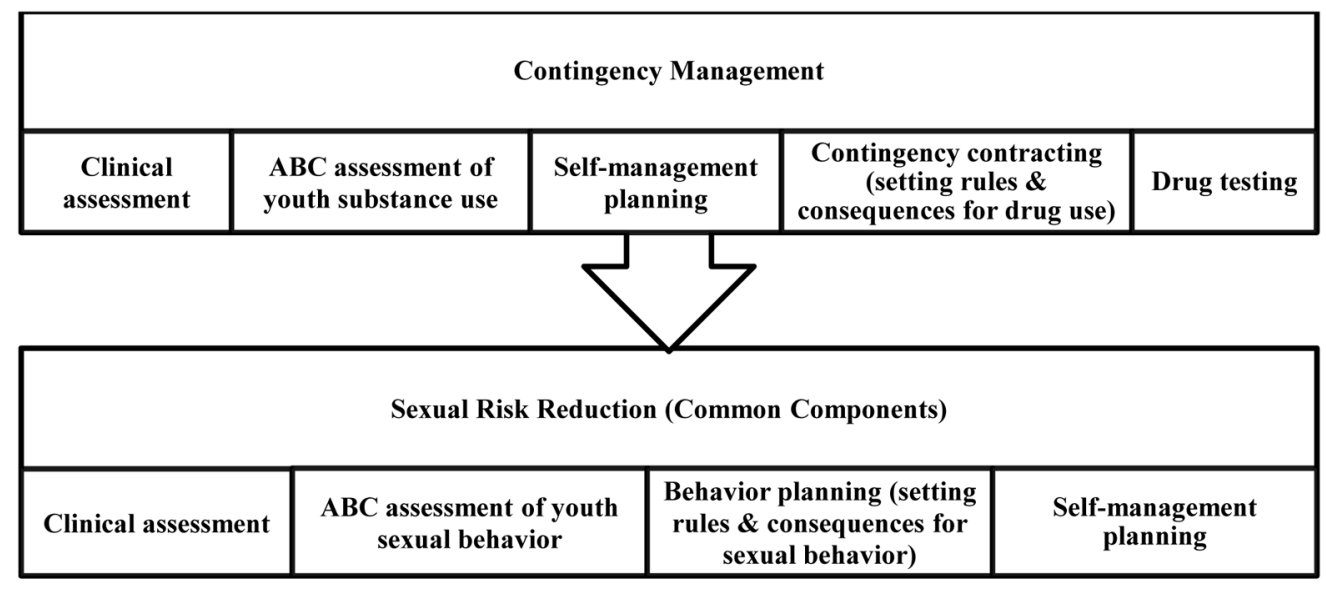

\begin{tabular}{|c|c|c|c|}
\hline \multicolumn{3}{|c|}{ Sexual Risk Reduction (Unique Components) } \\
\hline $\begin{array}{c}\text { Education about sexual } \\
\text { risks and safety }\end{array}$ & $\begin{array}{c}\text { Referral for HIV/STI } \\
\text { testing }\end{array}$ & $\begin{array}{c}\text { Condom use skill } \\
\text { training }\end{array}$ & $\begin{array}{c}\text { Obtaining birth } \\
\text { control }\end{array}$ \\
\hline
\end{tabular}

Figure 1.

Risk Reduction Therapy for Adolescents (RRTA) Core Components 


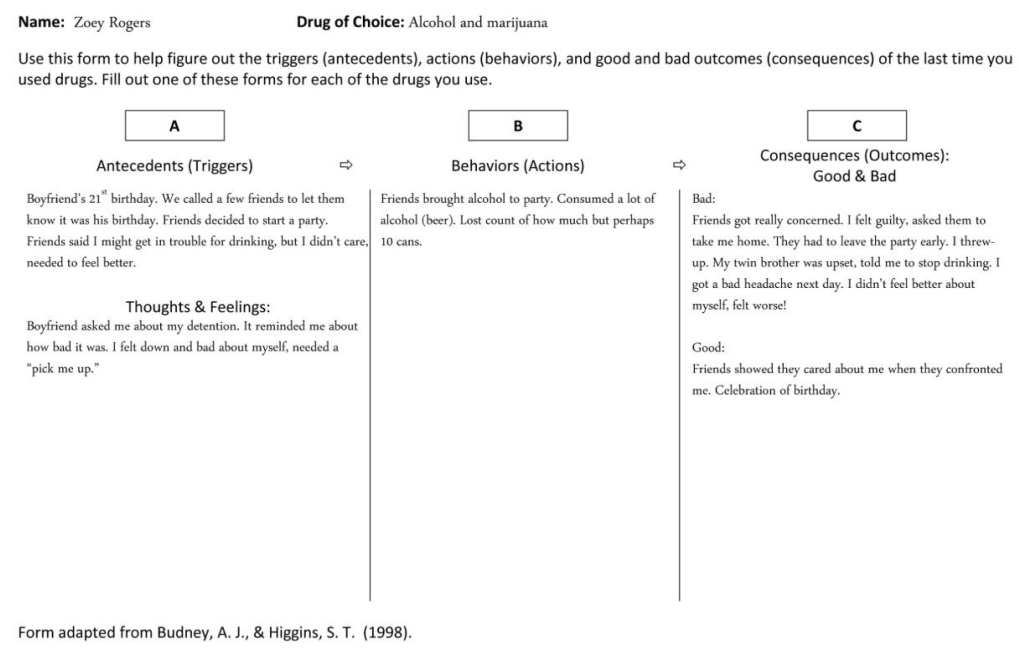

Figure 2.

Example antecedent-behavior-consequence (ABC) assessment form for substance use from the Risk Reduction Therapy for Adolescents protocol. 


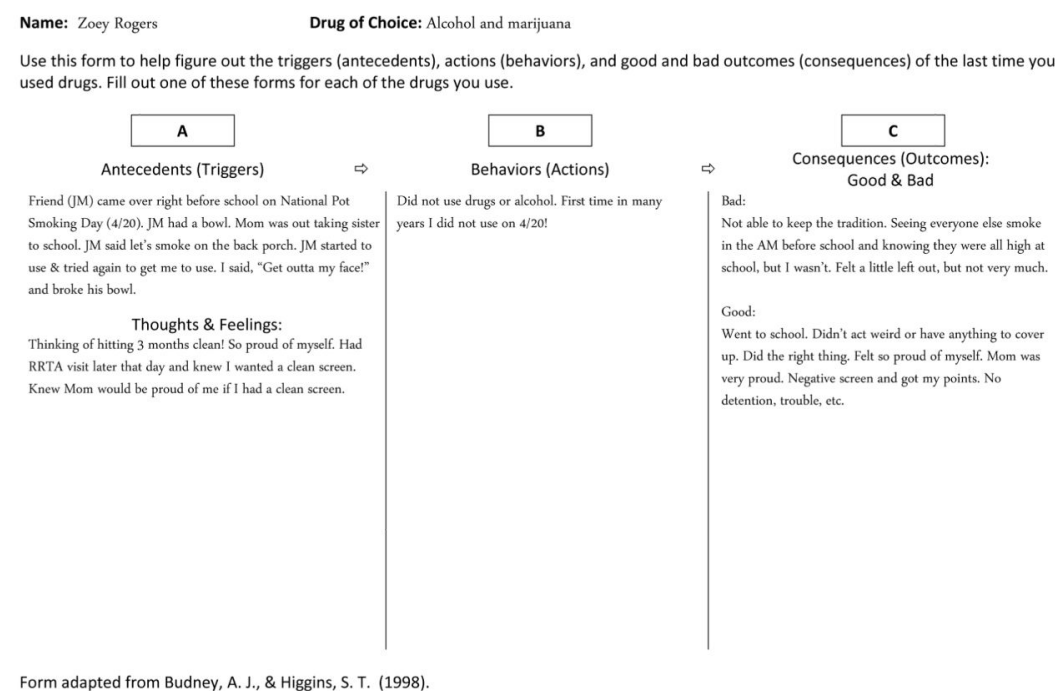

Figure 3.

Example antecedent-behavior-consequence $(\mathrm{ABC})$ assessment form for avoidance of substance use from the Risk Reduction Therapy for Adolescents protocol. 
Name: Zoey Rogers

Trigger: I'm with someone I trust (ex. Boyfriend, boy l've known for a long time)

Use this form to help create ways to manage the triggers for unprotected sex. This will help reduce the chances that triggers will lead to unprotected sex. Fill out one form for each trigger.

\begin{tabular}{|c|c|c|c|}
\hline \multirow[t]{2}{*}{ Plan } & \multicolumn{2}{|c|}{ Cost/Benefit Analysis } & \multirow{2}{*}{$\begin{array}{l}\text { Difficulty } \\
\text { (1-10 scale; } \\
10=\text { most } \\
\text { difficult) }\end{array}$} \\
\hline & Costs & Benefits & \\
\hline $\begin{array}{l}\text { A) } \\
\text { Always have TWO condoms } \\
\text { with me in my purse }\end{array}$ & $\begin{array}{l}\text { - They could break } \\
\text { - May not always have my purse with } \\
\text { me }\end{array}$ & $\begin{array}{l}\text { - Don't have to use them } \\
\text { - I have them on me } \\
\text { - More likely to use them }\end{array}$ & 3 \\
\hline $\begin{array}{l}\text { B) } \\
\text { Use condoms no matter what }\end{array}$ & $\begin{array}{l}\text { - Sex feels better w/o condom } \\
\text { - Partner might look down on me and } \\
\text { say something bad } \\
\text { - Partner may not want to have sex }\end{array}$ & $\begin{array}{l}\text { - Less likely to get an STI } \\
\text { - Less likely to get pregnant } \\
\text { - I'm taking responsibility and control } \\
\text { - No worried feelings }\end{array}$ & 2 \\
\hline $\begin{array}{l}\text { C) } \\
\text { Don't have sex }\end{array}$ & $\begin{array}{l}\text { - People might call me a prude } \\
\text { - My girlfriends won't care one way or } \\
\text { the other }\end{array}$ & $\begin{array}{l}\text { - Won't get pregnant } \\
\text { - No STIs! } \\
\text { - Guys won't "look down" on you for } \\
\text { using condoms }\end{array}$ & 1 \\
\hline
\end{tabular}

Figure 4.

Example self-management plan for safer sexual behavior from the Risk Reduction Therapy for Adolescents protocol. 


\section{Table 1}

\section{Expectations and Rules Related to Sex and Dating}

\section{Expectations reported by Zoey:}

1 Always have the bedroom door open if a male friend is in my bedroom

2 Mother must be home if I have a friend over

3 No dating anyone more than 3 years older than me

4 People I date must be in school and/or employed

5 I can't have sexual intercourse in our house

6 I need to be on birth control and use it properly

7 Be home by 7:30 pm on school nights

8 Mother needs to meet anyone I want to date before the first date

\section{Expectations reported by Ms. Rogers:}

1 Be in the house by 7:30 pm on school nights

2 People she dates must be in school and/or employed

3 People she dates cannot be more than 3 years older than her

4 I must meet and approve anyone she wants to date before the first date

\section{Rules in the behavior plan:}

1 Zoey must be in the house by 7:30 pm on school nights and 9:00 pm on weekend nights. If Zoey successfully completes treatment and probation, these will change to 9:00 pm on school nights and 11:00 pm on weekend nights (so long as Zoey is still following other behavior plan rules).

2 Zoey can only go on dates with people who are in school and/or employed.

3 Zoey cannot go on dates with anyone who is more than 3 years older than her.

4 If Zoey wants to go on a date, Mom must meet in-person and approve of the boy prior to the first date. Also, Zoey must not have sex with anyone Mom does not know.

5 If Zoey wants to have friends or boys come over to the house, Zoey must get approval from Mom and must leave the bedroom door wide open (door touching the back wall) if any boys are in Zoey's bedroom.

6 Zoey can't have sexual intercourse in our house or property.

7 If Zoey is having sex, she must be on birth control and use it correctly, and she must use condoms whenever she has sex. 
Table 2

Engagement of Families Who Have Received RRTA $(n=50)$

\begin{tabular}{lcccc}
\hline & High & Medium & Low & No \\
\hline Youth engagement & $47 \%$ & $25 \%$ & $26 \%$ & $2 \%$ \\
Parent engagement & $54 \%$ & $23 \%$ & $19 \%$ & $4 \%$ \\
Missed sessions & & & & \\
$\quad$ Mode & 1 & & & \\
$\quad$ Median & 2 & & & \\
\hline
\end{tabular}


